\title{
Editoriale del Dossier "Il contrasto alla corruzione come strumento di lotta politica"
}

\author{
Editorial do dossiê "A persecução da corrupção \\ como instrumento de luta política"
}

Editorial of dossier "The criminal prosecution of
corruption as an instrument of political struggle"

Renzo Orlandi ${ }^{1}$

University of Bologna, Italy

renzo.orlandi@unibo.it

https://orcid.org/0000-0002-3027-4972

Bruna Capparelli²

Autonomous University of Lisbon, Portugal

bruna.capparelli2@unibo.it

http://orcid.org/0000-0003-1249-2658

\begin{abstract}
Tradizionalmente, secondo la grammatica dei sistemi politicocostituzionali moderni di civil law, i luoghi della politica sono Parlamento e governo; magistratura e corti sono rette da altri principi, quelli del merito accertato in maniera imparziale e a condizioni di eguaglianza. Da una parte principio elettivo, dall'altra principio selettivo. I giudici sono in relazione con la politica solo indirettamente, per il tramite della legge, alla quale
\end{abstract}

1 Full Professor of Criminal Procedure - University of Bologna (Italy).

2 (2020-) Assistant Professor (Senior) of Criminal Procedure - Autonomous University of Lisbon. (2020-) Full Researcher of Criminal Procedure - "Ratio Legis" Autonomous University of Lisbon. (2018-2020) Researcher Associate of Criminal Procedure - "Ratio Legis" Autonomous University of Lisbon. (2018) PhD in Criminal Procedure - University of Bologna. (2018) PhD in Criminal Science PUCRS (joint doctorate). Bachelor's in Law - University of Bologna. Fully qualified attorney (Italy). 
detto corpo deve obbedire. Parlamento e governo sono i luoghi della democrazia, l'altro è quello dei migliori, o dei tecnici, o della competenza. Nondimeno, dogmi e concetti che reggevano una volta la distinzione tra ciò che pertiene alla magistratura e ciò che, invece, sfugge al suo dominio divengono a loro volta sfuggenti, e per superare molte difficoltà che derivano da queste ambiguità, si fa ormai silenziosamente capo a una figura meticcia, quella del "magistrato politico".

Passando per il mito dell'"uomo forte", alimentato dallo spirito antielitista e dal risentimento sociale, fino ad arrivare all'attuale assenza di gravitas nella gestione di decisioni collettive, ai modi di formazione dell'opinione pubblica e della sua influenza sulle decisioni politiche, con il presente editoriale gli Autori riflettono sulle cornici interpretative delle trasformazioni che stiamo vivendo - ampiamente discusse nell'ordinamento processuale penale italiano - nell'intento di stimolare il dibattito sul tema della "lotta alla corruzione", sia in una prospettiva di carattere generale, sia, più specificamente, nei suoi rapporti con il principio di legalità penale.

Parole Chiave: Editoriale; contrasto alla corruzione; diritto processuale penale; politica; "magistrato politico"; giustizia.

Resumo: Tradicionalmente, segundo a gramática dos sistemas políticosconstitucionais modernos de civil law, os locais da política são o Parlamento e o governo; o Judiciário e o Ministério Público são regidos por outros princípios, os do mérito apurados com imparcialidade e em condições de igualdade. De um lado, o princípio eletivo, de outro, o princípio seletivo. Os juízes e os promotores se relacionam com a política apenas indiretamente, por meio da lei, a que Judiciário e MP devem obedecer. Parlamento e governo são os lugares da democracia, o outro é o local dos melhores, ou dos técnicos, ou da competência. No entanto, dogmas e conceitos que antes mantinham a distinção entre o que pertence ao judiciário e ao Ministério Público e o que, por outro lado, escapa ao seu domínio, tornam-se por sua vez elusivos e, para superar muitas dificuldades que derivam dessas ambiguidades, criou-se silenciosamente uma figura híbrida, a do "magistrado político" (o que, no sistema italiano, abrange a figura do juiz e do promotor).

Passando pelo mito do "homem forte", alimentado pelo espírito antielitista e pelo ressentimento social, até a atual ausência de gravitas na gestão das decisões coletivas, nas modalidades de formação da opinião pública e sua influência nas decisões políticas, neste editorial pretende-se refletir sobre os marcos interpretativos das transformações que estamos vivenciando amplamente discutidas no sistema processual penal italiano - com o objetivo de estimular o debate sobre o tema da "luta contra a corrupção", tanto em 
uma perspectiva geral, quanto, mais especificamente, em suas relações com o princípio de legalidade penal.

Palavras-chave: Editorial; persecução da corrupção, Direito Processual penal; política; "magistrado político"; justiça.

ABSTRACT: Usually, according to the grammar of the modern politicalconstitutional systems of civil law, the places of politics are the Parliament and the government; judges and courts are governed by other principles, those of merit ascertained impartially and under conditions of equality. On the one hand, the elective principle, on the other, the selective principle. Judges are connected to politics only indirectly, through the law, which the Judiciary must obey. Parliament and government are the places of democracy, the other is the home of the best, or of technicians, or of competence. However, dogmas and concepts that once signaled the distinction between what pertains to the judiciary and what, on the other hand, escapes its dominion, become in turn elusive, and to overcome many difficulties that derive from these ambiguities, it is now silently headed to a hybrid figure of the "political magistrate".

Passing through the myth of the "strong man", powered by the anti-elitist spirit and some social bitterness, up to the current absence of gravitas in the management of collective decisions, to the ways of forming public opinion and its influence on political decisions, in this editorial the Authors reflect on the interpretative frameworks of the transformations we are experiencing - widely discussed in the Italian criminal procedure system aiming to stimulate the debate on the theme of the "fight against corruption", both from a general perspective, and, more specifically, in its relations with the principle of criminal legality.

KeYwords: Editorial; criminal prosecution of corruption; Criminal Procedure; political; "political magistrate"; justice.

SOMmARIO: Introduzione. 1. II contrasto alla corruzione come strumento di lotta politica. 2. Quali prospettive?

\section{INTRODUZIONE}

«La corruzione è fenomeno che trascende la questione penale. Sembra essere diventata una piaga politico-sociale, con significative ripercussioni anche sui bilanci economici degli Stati. In vari Paesi, i processi per fatti di 
corruzione hanno contribuito al tramonto di esperienze politiche [...] o di personalità di primissimo piano [...]. Si ha l'impressione che i protagonisti della politica contemporanea vivano sotto il costante sospetto di essere dei corrotti. [...] Di qui la tendenza degli ordinamenti processuali a superare l'ostacolo incoraggiando la collaborazione (whistleblowing, non punibilità, sconti di pena) o con particolari tecniche d'indagine (agenti provocatori, agenti sotto copertura). Per rendere più efficace il contrasto alla corruzione si istituiscono anche speciali unità investigative come accade ad esempio in Spagna con la Fiscalía Especial contra la Corrupción. In Italia è stata istituita una autorità amministrativa (Autorità Nazionale Anti-Corruzione) col compito di studiare e prevenire i fenomeni di corruzione, ma anche di raccogliere informazioni utili all'impostazione della attività repressiva delle procure della Repubblica $[. ..] »^{3}$ : alterazioni e distorsioni dello scenario odierno in tema di "corruzione" erano annunciate con queste parole nella scheda di presentazione della Call for Papers; si evidenziavano altresì le conseguenze politiche, giuridiche, economiche, finanziarie che da tempo connotano le politiche penali in tema di corruzione ${ }^{4}$, oltre alle fragilità sistematiche degli Stati-nazione, i quali, dovendosi adattare al nuovo contesto dominato dalla globalizzazione, lasciano sviluppare nel proprio interno autorità non statali per far fronte al fenomeno ${ }^{5}$. Bisogna pertanto saper diffidare del termine "corruzione" e dei sentimenti che evoca la "lotta" a un fenomeno certamente odioso, ma che si presta a

3 ORLANDI, Renzo. Il contrasto alla corruzione come strumento di lotta politica. In: Scheda di presentazione del dossier RBDPP 2019-2020, reperibile al link https://drive.google.com/file/d/1xnbo1wR-IrivgNnrfQ7McNrXkexZfvI3/view.

4 Considerazioni più tecniche sono contenute in CAPPARELLI, Bruna. Tutela dell'interesse finanziario europeo e processo penale interno. In: MARIA ISABEL ROMERO PRADAS, Integración Europea Y Justicia Penal: Nuevos Postulados, Retos Y Desafios De La Cooperación Judicial En La Unión Europea Homenaje A La Profesora Dra. Da Ma Isabel González Cano (Universidad De Sevilla). Tirant lo Blanck, 2020, p. 7 s.

5 Si crea così un'altra area di conflitti, perché attraverso le autorità indipendenti si sottrae al governo una parte dell'esecuzione e la si affida ad apparati indipendenti dal governo, operanti meno secondo criteri politici e più con tecniche e metodi di tipo quasi giudiziario. Ma a chi rispondono le autorità indipendenti? Quanto può estendersi il campo riservato alle autorità indipendenti? Paradossalmente, queste ultime, proprio per i motivi appena elencati, una volta istituite, non rischiano di subire un processo di erosione? 
facili strumentalizzazioni persino da parte di chi la corruzione la pratica sistematicamente, una volta occupate posizioni di potere. $E$ c'è poi da chiedersi cosa conosciamo sulla portata effettiva del fenomeno in questione nelle diverse aree del pianeta. Sappiamo se diminuisce o aumenta? Siamo in grado di misurare l'efficacia dei rimedi adottati e delle istituzioni (giudiziarie, amministrative) create per contrastarla?

Quanto al termine, esso comprende una varietà di reati, oltre che condotte che di per sé non sono vietate e sanzionate penalmente ma, semmai, da codici di condotta.

Rispetto alla portata, di certo occorre evitare di riferirsi alla corruzione "percepita", a sua volta influenzata da come e quanto i media riportano episodi e notizie, sicché, più frequenti sono le notizie (anche ripetute) di vicende corruttive e relative iniziative giudiziarie, più si ha l'impressione che il fenomeno sia diffuso; e più l'azione di contrasto è propagandata come efficace, maggiori sono le richieste di ulteriori interventi ${ }^{6}$. Meglio dunque riferirsi alla corruzione "sperimentata"

Per intendere come la corruzione influisca sulla politica, bisogna considerarne cause e costi.

Tra le cause, vanno menzionati assenza di professionalità nelle amministrazioni pubbliche, complessità e tempi dei processi di decisione, ricorso a procedure derogatorie: la prima perché indebolisce gli uffici pubblici, le altre perché conferiscono a essi un eccesso di discrezionalità.

6 Sappiamo pure che più l'area privata avanza nel perimetro statale, più l'attività pubblica presta il fianco alla corruzione. Questa costituisce un ulteriore limite perché modifica la corretta trasmissione degli impulsi popolari provenienti dalle assemblee rappresentative o dal governo: decisioni di spesa, allocazione delle risorse, localizzazione degli investimenti, distribuzione di benefici e altri tipi di decisioni vengono così deviati dalla loro corretta direzione. A tali propositi cfr. GALASSO, Giuseppe. Liberismo e democrazia, Salerno, 2013, p. 8 s.

7 In base agli indici di corruzione percepita, l'Italia è al sessantunesimo posto su 167 Paesi, dietro Stati come Namibia, il Ruanda e la Romania. Le due indagini di Eurobarometro e "Trasparency Internacional”, dirette ad accertare se gli intervistati fossero stati oggetto di richieste o aspettative di tangenti, mostrano invece che l'Italia si colloca nella media europea. Naturalmente, anche i dati sulla corruzione sperimentata vanno usati con cautela a causa delle domande rivolte agli intervistati e del campione prescelto. Il rapporto IstAT sulla corruzione è disponibile in www.istat.it: La corruzione in Italia: il punto di vista delle famiglie, 12 ottobre 2017. 
Quanto ai costi, i maggiori sono costituiti dal dispendio di denaro pubblico, dalle diseguaglianze che ne conseguono, a danno di coloro che si trovano "le porte chiuse", e dalla sfiducia prodotta nei cittadini rispetto alle istituzioni democratiche. Ai costi economici si aggiungono quelli politici, riguardanti il circuito della democrazia (sostegno dei propri rappresentanti, ai quali è affidato il compito di scegliere i governi, chiamati a loro volta a dirigere gli uffici pubblici, erogare servizi alla collettività). Se tale circuito si incrina, se lungo di esso si registrano perdite, il sostegno popolare diminuisce e nascono movimenti di ispirazione populistica, di ribellione, alimentati dalla sfiducia, con inevitabili pericoli per la sopravvivenza dell'apparato democratico.

Su queste problematiche sono stati invitati a riflettere - nello specifico dell'ordinamento italiano e non solo - gli autori del presente numero monografico. Aleggiavano sullo sfondo più ampie questioni, che hanno inevitabilmente guadagnato la scena dell'analisi critica: il rapporto tra diritto e politica, tra democrazia e diritto, tra legalità e giustizia ${ }^{8}$, tra "magistrato politico" e giustizia ${ }^{10}$.

\section{Il CONTRASTO ALLA CORRUZIONE COME STRUMENTO DI LOTTA POLITICA}

Per "lottare" contro la corruzione in Italia sono impegnati un'apposita Autorità nazionale (ANAC), che avrebbe compiti preventivi, e le procure, con compiti repressivi (su queste ultime, si ritornerà in seguito).

In particolare, l'ANAC, la cui azione è invocata con una funzione salvifica della giustizia, è nondimeno sovracaricata di molteplici funzioni (oltre all'anticorruzione: trasparenza, inconferibilità e incompatibilità degli incarichi, misure straordinarie nei confronti dell'impresa, controllo dei

8 Al riguardo si veda CAPPARELLI, Bruna. Il potere giudiziario e la legge processuale penale in Italia, in MONTEIRO GUEDES VALENTE (a cura di), Os desafios do direito do século XXI: genoma humano, europeísmo, poder e política, Constituição e democracia, Almedina, 2019, p. 101-133.

9 L'espressione è contenuta nella prefazione di Tullio Padovani al testo SGUBBI, Filippo. Il diritto penale totale, Bologna, 2019, p. 15.

10 Cfr. GUARNIERI, Carlo. Giustizia e politica: $i$ nodi della seconda repubblica, Bologna, 2003, p. 28 s. 
contratti pubblici, monitoraggio, codici di comportamento dei dipendenti pubblici ecc. ecc.), e poco attrezzata per svolgerle.

L'inefficacia dell'azione pubblica, prodotta anche dalla corruzione e dai rimedi predisposti per impedirla, produce un divario tra promesse e risultati, scaricando sulla "classe politica" gli effetti di attese a cui non corrispondono realizzazioni, con conseguenti frustrazioni e sfiducia nei confronti degli organismi rappresentativi.

Ma le decisioni prese dagli organi rappresentativi in nome del popolo sono, si sa, limitate dal diritto. Dunque, anche la sovranità popolare è sottoposta a vincoli, alcuni di questi sono particolarmente stretti. La sovranità popolare non può liberamente espandersi. Come è noto, la componente garantistica del potere pubblico, fondata sul diritto, sui giudici, sulle autorità indipendenti, sul metodo di decisione mediante contraddittorio (processo), viene storicamente prima della componente democratica, perché si è sviluppata grazie all'apporto del pensiero liberale del XVIII e del XIX secolo ${ }^{11}$. Sia perché ha tradizione più risalente, sia perché consiste principalmente di un metodo, questa componente si è estesa, penetrando tanto nell'elemento democratico quanto in quello autoritativo. Anche la legge, voluta dai rappresentati del popolo, è sottoposta a un giudice. Anche la decisione amministrativa, presa dal potere esecutivo, è sottoposta, all'occorrenza, a un giudice, che ne valuta la corrispondenza ai parametri legali. Ma il controllo da parte del giudice di leggi e di atti amministrativi è, poi, andato oltre, determinando continue tensioni. Dal parlamento, di tanto in tanto, provengono segni di insofferenza per la Corte costituzionale e per la magistratura in generale. L'opinione pubblica lamenta che ogni decisione, per le quali vigono ordinamenti separati e autonomi, facenti capo a enti con personalità giuridica propria, sui quali vigilano appositi tribunali, finisca quasi sempre dinnanzi a un Tribunale amministrativo. Si riaffaccia l'antico interrogativo: se la suprema autorità sia affidata al popolo o al diritto (avendo l'ultima parola, in questo secondo caso, i giudici). Ma la forza espansiva del diritto nel campo dell'autorità si manifesta anche in un altro modo, imponendo all'autorità di liberarsi dei suoi privilegi, di perseguire il fine pubblico

11 Per una lucida analisi del fenomeno v. CASSESE, Sabino. La crisi dello Stato, 2002, Laterza, p. 65 s. 
indicato dalla legge con mezzi non diversi da quelli privati. Le corti sono spesso accusate di fare politica. Ci si chiede se le decisioni dell'organo democratico per eccellenza, il legislatore (sia nazionale, sia locale), possano essere sottoposte allo scrutinio di un organo che opera come giudice neutrale, non influenzato dalla politica e dalle decisioni democratiche ${ }^{12}$. Gli organismi rappresentativi possono dettare norme ma non possono regolare casi concreti la cui decisione è rimessa a sistemi giudiziari. Da qui le continue tensioni tra politica e giustizia. Queste tensioni sono particolarmente accentuate quando i giudici affrontano problemi sociali e politici importanti, come quello della corruzione. In questi casi, essi hanno la scelta tra restraint e activism e seguono strategie molto diverse: rifiutarsi di decidere, dichiarando la questione inammissibile; posporre la decisione; definirla in termini generali; riconoscere un diritto e una procedura, lasciando autonomie nelle scelte concrete ecc. ecc. L'atteggiamento dei giudici è molto diverso. I giudici operano nella trama fissata dalla legge, ma non sono solo esecutori della legge. C'è una grande quantità di giudici che assumono un ruolo importante nel prendere decisioni, nel fare scelte. Molte decisioni, poi, non sono rilevanti solo per le parti in giudizio, ma anche per la collettività. È in ordine a queste che si verificano i più estesi contrasti tra politica, gli organi rappresentativi e i giudici.

Si registra, altresì, una crescente domanda di giustizia che scarica sul sistema giudiziario una grande quantità di istanze. Ma se i giudici ampliano la propria sfera d'azione e il peso delle loro decisioni, si verificano almeno due conseguenze: (1) l'offerta di giustizia crea una crescente domanda di giustizia; (2) il sistema giudiziario svolge una sempre maggiore surrogazione dei meccanismi democratici di decisione.

Come è noto, molti episodi di contrasto alla corruzione in Italia e nel mondo riportano i riflettori sulla figura del pubblico ministero. In Italia le procure della Repubblica sono dotate di indipendenza nei confronti degli organi politici. Svolgono il ruolo di accusa. Ma debordano sia invadendo campi estranei, sia utilizzando, attraverso l'uso distorto di intercettazioni

12 La diffusione universale di standard, come quello di proporzionalità, fa pensare a una globalizzazione della giustizia, confermata dal frequente dialogo tra i giudici, che accentua il limite per la democrazia prodotto dalla giustizia nazionale. Cfr. MANES, Vittorio. Il giudice nel labirinto. Profili delle intersezioni tra diritto penale e fonti sovranazionali, Dike, 2012, p. 45 s. 
e custodia cautelare, procedure di "naming and shaming", poco produttive sul piano processuale, ma molto efficaci sul piano politico e dell'opinione pubblica, spesso poste in essere al solo scopo di additarle al pubblico ludibrio. Come è facile intuire, gli effetti principali di questi interventi si producono nell'ambito politico, stimolando la sfiducia dell'elettorato, con la divulgazione di atti che dovrebbero essere noti solo alle parti, conditi di dettagli irrilevanti per il diritto ma rilevanti per il costume. Insomma, si nomina per svergognare. Le intercettazioni diventano un modo per fare processi in piazza ${ }^{13}$, come non si faceva neppure in età medioevale. Si ricorre con troppa facilità a strumenti che non richiedono neppure di installare mezzi di raccolta dati e conversazioni (una delle specie di trojan). Ci si fida del parlato, non dello scritto. Si ricorre a conversazioni tra terze persone per incolpare un soggetto estraneo alla conversazione. I dati raccolti diventano - in modi non accertati - di dominio pubblico e finiscono sulle prime pagine dei giornali. Alcune regole elementari sull'esercizio della funzione di giustizia sono abbandonate. Non c'è rispetto del criterio di proporzionalità (era possibile ricorrere a mezzi meno invasivi?). Non è rispettato il principio costituzionale della comunicazione riservata delle accuse all'incolpato. Si dimentica il principio di cautela nell'esercizio di compiti che attengono alla vita, alla libertà, alla riservatezza. In questa direzione, i procuratori sono sempre più proiettati nello spazio pubblico, dove sono ascoltati più per i poteri di cui dispongono che per quello che pensano, e conseguentemente diventano i naturali candidati alle posizioni di vertice di quella politica della quale dovrebbero restare distanti per dovere d'ufficio.

Di fatto, negli ordinamenti moderni le procure svolgono una pluralità di ruoli, supplendo o sostituendo decisioni che dovrebbero essere collettive, in nome di una indipendenza che viene tradita dal loro dialogo diretto con l'opinione pubblica e dalle carriere politiche che ne conseguono per i singoli procuratori.

I conflitti con il corpo politico in Italia sono stati noti e numerosi: alcuni fisiologici, perché la giustizia costituisce un naturale limite della

13 Per riflessioni sulle modalità di impostazione dell'indagine da parte del pool milanese durante Tangentopoli, v. ORLANDI, Renzo. Inchieste preparatorie nei procedimenti di criminalità organizzata: una riedizione dell'inquisitio generalis?, Riv. it. dir. e proc. pen. 1996, p. 555 s. 
politica; altri patologi, perché la giustizia non risponde al compito fondamentale che è chiamata a svolgere.

Se ci si muove da questo piano teorico, da questi grandi problemi, e si passa al livello applicato ${ }^{14}$, alle pratiche, il quadro si presenta assai più problematico. Ma anziché ricordare singoli episodi sarebbe preferibile avviare una riflessione sui cambiamenti strutturali dell'ordine giudiziario. Questo si è sdoppiato, e non nel senso indicato da coloro che invocano la separazione delle carriere. Sembra quasi che le procure non siano più in funzione del giudizio, ma accusano e giudicano. E, anzi, spesso non sembrano interessate a vincere nel processo, dinnanzi a un giudice terzo, come dimostrano le posizioni di chi vorrebbe un sistema in cui l'imputato è costretto a patteggiare prima ancora del processo. Una condanna senza giudice, irrogata direttamente dall'ufficio dell'accusa. Il filtro che doveva essere svolto dal giudice per le indagini preliminari, nei confronti dell'azione dei pubblici ministeri, è venuto meno, per la debolezza funzionale del Gip. Come si ricorderà, con il vecchio codice di procedura penale, il giudice istruttore era il vero dominus dell'istruttoria, che era da lui condotta e che si concludeva con un'ordinanza, da lui sottoscritta, di rinvio a giudizio o di proscioglimento ${ }^{15}$. Il giudice istruttore svolgeva una vera e propria dialettica con il pubblico ministero. Nel sistema attuale, il vasto materiale raccolto dai pubblici ministeri viene inviato al giudice per le indagini preliminari che, per ragioni strutturali e per la funzione residuale da loro svolta, si limita, di fatto, a un controllo formale e "cartaceo". Tutto finisce nel dibattimento, che secondo il codice del 1988, come è noto, è il luogo in cui si forma la prova. Inoltre, sempre secondo il "nuovo" codice di procedura penale, i processi si sarebbero dovuti svolgere in gran parte secondo i riti alternativi (giudizio abbreviato e applicazione della pena su richiesta delle parti). Così non

$14 \mathrm{~V}$, per tutti, ORLANDI, Renzo. Diritti individuali e processo penale nell'Italia repubblicana, in NEGRI, Daniele e PIFFERI, Michele (a cura di), Diritti individuali e processo penale nell'Italia repubblicana. Materiali dell'incontro di studio Ferrara 12-13 dicembre 2010, Giuffrè, 2011, p. 51-83.

15 Cfr. CONSO, Giovanni. Precedenti storici e iter della legge n. 108 del 1974, in CONSO G., GREVI V., NEPPI MODONA G., Il nuovo codice di procedura penale. Dalle leggi delega ai decreti delegati, I, La legge delega del 1974 e il progetto preliminare del 1978, Cedam, 1989, p. 3-75. 
è stato. Il potere delle procure è quindi cresciuto negli interstizi delle procedure. E questa disfunzione può dirsi ulteriormente aggravata con l'eccessiva durata dei processi. Se il processo fosse più rapido, la verifica dell'azione delle procure sarebbe tempestiva, e il loro potere, anche in relazione ai mezzi di comunicazione di massa, sarebbe condizionato dalla sollecitudine del giudizio. Quando il processo è rapido, la dialettica tra le parti e tra le diverse posizioni, anche nell'opinione pubblica, è più equilibrata. Quando, al contrario, dopo un'incisiva azione delle procure (arresti, intercettazioni rese pubbliche e perquisizioni), il dibattimento in primo grado si svolge a distanza di anni, l'accusatore diventa giudice, perché il giudizio definitivo arriverà troppo tardi ${ }^{16}$.

Tornando allo scambio di ruoli tra giudice e procure, è utile ricordare come ci sono ancora molte componenti che andrebbero studiate attentamente, che richiedono analisi sia giuridiche, sia sociologiche. Il trapasso delle procure da organo di accusa a organo di accusa-giudizio avviene in virtù dell'azione combinata di più protagonisti, i cui legami non sono sempre evidenti. Il primo passo lo fanno le procure che "svelano"; il secondo i mezzi di comunicazione, che diffondono; il terzo le forze politiche che sanzionano. Una specie di azione coordinata tra poteri dello Stato, opinione pubblica e forze politiche, che trova corrispondenza nella richiesta dello Stato verso la società, di assenza di impermeabilità dell'azione pubblica, di trasparenza. A tal proposito, è sufficiente ricordare il modo efficace in cui vengono denominate le inchieste, a opera delle autorità di polizia e delle procure, e la tecnica con la quale vengono gestite le comunicazioni successive, in modo da assorbire e superare le reazioni degli indagati che vedono i propri nomi sui giornali; oppure al rilievo dato alle indagini delle procure, comparato con il nessun rilievo che hanno sui mezzi di comunicazione le sentenze emanate al termine dei processi ${ }^{17}$. Si tratta di un nuovo potere, che si consolida attraverso la narrazione di un Italia corrotta e che avvalora una visione negativa

16 Cfr. BRONZO, Pasquale. La ragionevole durata sotto l'incubo prescrizione, in Sistema Penale, 29 luglio 2020 e MAZZA, Oliviero. La riforma della prescrizione dei due orologi: la prescrizione fra miti populistici e realtà costituzionale, in Sistema Penale, 21 gennaio 2020.

17 A tal proposito, si ricordano le parole pronunciate - «cronaca giudiziaria come mezzo di lotta politica» - dal procuratore della repubblica Giuseppe Pignatone 
della società italiana. Il passaggio successivo è inevitabile: la politica si impossessa dell'accusa-giudizio, e, a sua volta, diventa il nuovo eroe popolare, un generoso giustiziere che ruba all'élite per dare al popolo, giacché «dal popolo viene la forza» ${ }^{18}$.

\section{Quali PROSPETtive?}

L'analisi appena esposta è incompleta e sommariamente abbozzata, né questa era la sede per entrare in più sottili dettagli. La prospettiva illustrata si presta tuttavia a essere adottata come ipotesi di lavoro.

In ogni caso, ciò che emerge è che sembra essersi venuto a creare un nuovo potere dello Stato, che accusa e giudica in piazza, che è dotato di un potere sanzionatorio informale, ma efficace. Proprio grazie a esso, politica e giustizia ristabiliscono un antico legame, come, del resto, hanno notato gli studiosi francesi che parlano oramai di "Stato della giustizia"19, anziché di Stato di diritto.

In questo quadro delineato, i giudici, paradossalmente, finiscono col passare in secondo piano e la presunzione d'innocenza sembra scomparire del tutto ${ }^{20}$.

Più in generale, i poteri pubblici sono fabbricati con materiali diversi, con consistenze e resistenze differenti che sono obbligati a legare tra di loro. Da tutti questi materiali essi traggono una fonte di legittimazione: sono legittimati dal popolo, dal diritto, dall'effettività delle decisioni. Nessuno di questi elementi può mancare. Nondimeno, tutti restano assieme con difficoltà, perché il potere pubblico risponde a esigenze contraddittorie. Occorre dunque ristabilire una zona di

in occasione dell'incontro di studi all'Università Luiss Guido Carli dal titolo Il rischio reputazionale nell'era dei social media, Roma, 21 ottobre 2019.

La frase si può leggere in RIVAROL, Antonie. Esprit de Rivarol, Paris, 1808, p. 37.

19 Per tutti, v. KRYNEM, Jacques. L'emprise contemporaine des judges, Paris, 2012.

20 Ancora estremamente attuali le osservazioni contenute in ILLUMINATI, Giulio. La presunzione d'innocenza dell'imputato, Zanichelli, 1979. 
equilibrio $^{21}$. Si può legittimamente chiedere che la magistratura requirente proceda con quella cautela che è propria di un magistrato. Altrimenti, si dà ragione a chi sostiene che, dei poteri dello stato, l'unico che non risponde a nessuno è la magistratura; a chi ritiene che le procure seguano propri indirizzi politici, cercando di influenzare l'opinione pubblica o - peggio - a chi ritiene che esse si facciano strumentalizzare da forze o orientamenti politici esterni ovvero che il CSM sia diventato uno strumento di trasmissione di pressioni interne, di corrente, oppure personali, con collegamento con figure appartenenti al mondo dei partiti, non meritando così l'indipendenza assicurata dall'ordinamento all'ordine giudiziario nel suo complesso. In particolare, colpiscono alcune circostanze che mostrano assenza di rispetto per le istituzioni e di regole deontologiche elementari: colloqui su argomenti ufficiali, come la scelta di titolari di cariche pubbliche, che si svolgono al ristorante anziché in ufficio; partecipazione a tali colloqui di soggetti estranei al CSM; ricorso ad argomenti estranei (persone indicate "perché dei nostri" e non per specifiche qualità individuali o per esigenze legate alla carica); decisioni "a pacchetto", che si sostanziano nella scelta di più persone insieme per accontentare tutti o, ancora, inchieste pilotate e interferenza nella selezione del personale con procedure d'ufficio delle procure, ossia esattamente la "stortura" per evitare la quale era stato istituito il CSM. Insomma, si tratta indubbiamente di metodi molto irregolari.

In definitiva, si potrebbe affermare che l'ordine giudiziario registra attualmente gravi inefficienze e che al suo interno si muovono soggetti che non rispondono agli alti standard richiesti a un magistrato. Anzi, le ferite alla legittimità sono inferte proprio da coloro che dovrebbero assicurarla, alimentando l'anti-Stato. Ma se chi fa politica non ha una politica, ecco che il vuoto è inevitabilmente riempito dalle procure e da questa nuova figura del "magistrato politico".

21 Da un lato, il Parlamento esonda con leggi sempre più dettagliate, che aspirano a essere auto-applicative, trasformandosi in legislatore-amministratore. Dall'altro lato, le decisioni collettive sono sempre più nelle mani delle procure e dei giudici. La prima tendenza espande la democrazia, la seconda amplia l'epistocrazia. Ambedue limitano l'azione dell'amministrazione, per cui l'esecutivo viene privato della sua discrezionalità e le competenze tecniche degli uffici vengono frustrate. 


\section{Bibliografia}

BRONZO, Pasquale. La ragionevole durata sotto l'incubo della prescrizione, in Sistema Penale, 29 luglio 2020.

CAPPARELLI, Bruna. Il potere giudiziario e la legge processuale penale in Italia, in MONTEIRO GUEDES VALENTE (a cura di), Os desafios do direito do século XXI: genoma humano, europeísmo, poder e política, Constituição e democracia, Almedina, 2019, p. 101-133.

CAPPARELLI, Bruna. Tutela dell'interesse finanziario europeo e processo penale interno, in MARIA ISABEL ROMERO PRADAS, Integración Europea Y Justicia Penal: Nuevos Postulados, Retos Y Desafios De La Cooperación Judicial En La Unión Europea - Homenaje A La Profesora Dra. Da Ma Isabel González Cano (Universidad De Sevilla). Tirant lo Blanck, 2020, p. 1-35.

CASSESE, Sabino. La crisi dello Stato, 2002, Laterza, p. 65 s.

CONSO, Giovanni. Precedenti storici e iter della legge n. 108 del 1974, in CONSO G., GREVI V., NEPPI MODONA G., Il nuovo codice di procedura penale. Dalle leggi delega ai decreti delegati, I, La legge delega del 1974 e il progetto preliminare del 1978, Cedam, 1989, p. 3-75.

GALASSO, Giuseppe. Liberismo e democrazia, Salerno, 2013, p. 8 s.

GUARNIERI, Carlo. Giustizia e politica: i nodi della seconda repubblica, Bologna, 2003, p. 28 s.

ILLUMINATI, Giulio. La presunzione d'innocenza dell'imputato, Zanichelli, 1979.

KRYNEM, Jacques. L'emprise contemporaine des judges, Paris, 2012.

MANES, Vittorio. Il giudice nel labirinto. Profili delle intersezioni tra diritto penale e fonti sovranazionali, Dike, 2012, p. 45. s.

MAZZA, Oliviero. La riforma della prescrizione dei due orologi: la prescrizione fra miti populistici e realtà costituzionale, in Sistema Penale, 21 gennaio 2020.

ORLANDI, Renzo. Il contrasto alla corruzione come strumento di lotta politica, Scheda di presentazione del dossier RBDPP 2019-2020, reperibile al link https://drive. google.com/file/d/1xnbo1wR-IrivgNnrfQ7McNrXkexZfvI3/view.

ORLANDI, Renzo. Diritti individuali e processo penale nell'Italia repubblicana, in NEGRI, Daniele e PIFFERI, Michele (a cura di), Diritti individuali e processo penale nell'Italia repubblicana. Materiali dell'incontro di studio Ferrara 12-13 dicembre 2010, Giuffrè, 2011, p. 51-83. 
ORLANDI, Renzo. Inchieste preparatorie nei procedimenti di criminalità organizzata: una riedizione dell'inquisitio generalis?, Riv. it. dir. e proc. pen. 1996, p. 555 s. RIVAROL, Antonie. Esprit de Rivarol, Paris, 1808.

SGUBBI, Filippo. Il diritto penale totale, Bologna, 2019.

\section{Informações adicionais e declarações do autor (integridade científica)}

Acknowledgment: Gli Autori ringraziano i dott. Mattia Giangreco e Vinicius Vasconcellos per l'attento lavoro di revisione del testo dello scritto.

Conflict of interest declaration: the authors confirm that there are no conflicts of interest in conducting this research and writing this article.

Declaration of authorship: all and only researchers who comply the authorship requirements of this article are listed as authors; all coauthors are fully responsible for this work in its entirety.

- Renzo Orlandi: conceptualization, methodology, data curation, investigation, writing - original draft, validation, writing - review and editing, final version approval.

- Bruna Capparelli: conceptualization, methodology, data curation, investigation, writing - original draft, validation, writing - review and editing, final version approval.

Declaration of originality: the authors assure that the text here published has not been previously published in any other resource and that future republication will only take place with the express indication of the reference of this original publication; they also attest that there is no third party plagiarism or self-plagiarism. 


\section{COMO CITAR ESTE EDITORIAL:}

ORLANDI, Renzo; CAPPARELLI, Bruna. Editoriale del dossier "II contrasto alla corruzione come strumento di lotta politica". Revista Brasileira de Direito Processual Penal, Porto Alegre, vol. 6, n. 3, p. 1119-1134, set./dez. 2020. https://doi.org/10.22197/rbdpp.v6i3.458

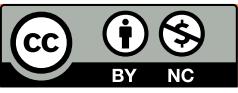

Esta obra está licenciada com uma Licença Creative Commons Atribuição-NãoComercial 4.0 Internacional. 